\title{
Criminologie
}

\section{L'intervention par les pairs : un outil pour soutenir la sortie de rue}

\section{Céline Bellot, Jacinthe Rivard et Élisabeth Greissler}

Volume 43, numéro 1, printemps-été 2010

Les jeunes et la rue

URI : https://id.erudit.org/iderudit/044056ar

DOI : https://doi.org/10.7202/044056ar

Aller au sommaire du numéro

\section{Éditeur(s)}

Les Presses de l'Université de Montréal

ISSN

0316-0041 (imprimé)

1492-1367 (numérique)

Découvrir la revue

Citer cet article

Bellot, C., Rivard, J. \& Greissler, É. (2010). L’intervention par les pairs : un outil pour soutenir la sortie de rue. Criminologie, 43(1), 171-198.

https://doi.org/10.7202/044056ar
Résumé de l'article

La question de la "sortie de rue » des jeunes est peu abordée dans les écrits scientifiques. Dans cet article, nous avons souhaité l'aborder à partir d'un regard croisé sur deux recherches que nous avons conduites autour du Collectif d'intervention par les pairs. Il s'agit de saisir ici les apports de ce cadre d'intervention dans la trajectoire des jeunes. La participation de jeunes ayant vécu dans la rue à cette intervention les place dans un nouveau rôle : elle représente pour eux à la fois une occasion de faire l'expérience de distanciation et de proximité du monde de la rue dans un cadre différent et une réelle opportunité d'engagement dans le monde conventionnel de l'intervention. Pourtant, que dire, au-delà de cette participation, des enjeux de reconnaissance et de non-reconnaissance que vivent les jeunes en situation de rue? 


\title{
L'intervention par les pairs: un outil pour soutenir la sortie de rue
}

\author{
Céline Bellot \\ École de service social, Université de Montréal \\ celine.bellot@umontreal.ca \\ Jacinthe Rivard \\ Coordonnatrice de la recherche évaluative \\ Collectif d'intervention par les pairs \\ jacinthe.rivard@umontreal.ca \\ Élisabeth Greissler \\ Étudiante au doctorat \\ École de service social, Université de Montréal \\ elisabeth.greissler@umontreal.ca
}

RÉSUMÉ - La question de la "sortie de rue» des jeunes est peu abordée dans les écrits scientifiques. Dans cet article, nous avons souhaité l'aborder à partir d'un regard croisé sur deux recherches que nous avons conduites autour du Collectif d'intervention par les pairs. Il s'agit de saisir ici les apports de ce cadre d'intervention dans la trajectoire des jeunes. La participation de jeunes ayant vécu dans la rue à cette intervention les place dans un nouveau rôle: elle représente pour eux à la fois une occasion de faire l'expérience de distanciation et de proximité du monde de la rue dans un cadre différent et une réelle opportunité d'engagement dans le monde conventionnel de l'intervention. Pourtant, que dire, au-delà de cette participation, des enjeux de reconnaissance et de non-reconnaissance que vivent les jeunes en situation de rue?

MOTS CLÉs - Jeunes de la rue, sortie de rue, intervention, pairs-aidants, trajectoires, identité, déviance/marginalité.

\section{Introduction}

L'influence des pairs est généralement construite de manière négative en criminologie. Les pairs seraient en effet un des vecteurs de l'engagement des jeunes dans des comportements déviants, voire délictueux 
(Morizot et Le Blanc, 2000; Lanctôt et al., 2007; Baron, 2008). Pourtant, dans d'autres disciplines, l'influence des pairs est construite autrement. Ainsi, en santé publique, en éducation, en service social, les pairs et leur influence sont les moteurs de stratégies d'intervention qui visent à promouvoir un rôle de modèle en termes de comportements et d'attitudes, y compris lorsque des jeunes ont adopté des comportements déviants et délictueux (Milburn, 1995; Sciacca et al., 1996; Backett et al., 2000; Fontaine et al., 2006; Proulx et al., 2006; Bellot et al., 2007). C'est au cœur de ce paradoxe d'influences positives ou négatives que se situe notre article. Il s'agit en effet, à partir de deux recherches conduites en partie autour d'un dispositif d'intervention, le Collectif d'intervention par les pairs, de montrer comment les stratégies d'intervention par les pairs donnent une opportunité de reconnaissance d'une expérience de la rue qui peut alimenter le processus de sortie de rue des jeunes, tout en fournissant un cadre d'influence susceptible de soutenir et d'aider les jeunes qui sont encore dans la rue. Les jeunes dont il est question ici sont des jeunes qui ont vécu dans la rue pour une période de plus de trois jours (critère employé dans la plupart des études). En ce sens, tous les pairs-aidants ont, de notre point de vue et du point de vue du Collectif, une expérience de rue, c'est-à-dire un vécu dans les rues de Montréal qui leur a permis de tisser des relations, d'avoir des activités routinières, de développer une identité. Ainsi, une relecture de ces recherches déjà réalisées permet d'analyser comment la reconnaissance de l'expérience de la rue, à travers une intervention par les pairs, peut soutenir une forme de réinsertion socioprofessionnelle des pairs-aidants, sans que celle-ci ait été visée.

Dans un premier temps, une recherche évaluative portant sur le dispositif d'intervention par les pairs nous a permis de constater l'effet sur la trajectoire des pairs eux-mêmes, de leur expérience dans le Collectif, tout comme sur les jeunes de la rue, cibles de l'intervention ${ }^{1}$.

1. Cette recherche évaluative du dispositif a été conduite sous la direction de Céline Bellot et de Céline Mercier, de 2002 à 2004. Financée par le CQRS (ancien FQRSC), l'étude a eu pour objectif de décrire les effets de l'intervention tant auprès des jeunes de la rue, des pairs, que des partenaires et de la communauté. Elle a aussi visé à décrire de manière plus approfondie les multiples niveaux et logiques d'action au cœur de ce dispositif. Elle a permis notamment de montrer comment le déploiement d'un cadre alternatif, placé en tension entre la marginalité et la conventionnalité permet aux acteurs de mieux rejoindre et d'agir préventivement auprès des jeunes de la rue qui sont en rupture avec les stratégies habituelles d'intervention. Ainsi, les pairs-aidants, comme le dispositif lui-même, apparaissent comme un relais non menaçant entre la rue et le réseau habituel des services 
Dans un second temps, une recherche compréhensive portant sur les expériences de la sortie de rue a permis de rencontrer 18 pairs et anciens pairs ayant participé au Collectif ${ }^{2}$.

Sortir les jeunes de la rue constitue un impératif porté par la plupart des interventions et des politiques publiques dans les villes de nombreux pays. Ces interventions normatives tentent le plus souvent de diaboliser et d'effacer l'expérience de rue pour renforcer l'adoption d'un mode de vie qualifié de plus conventionnel et conforme aux attentes sociétales. Le Collectif d'intervention par les pairs du centre-ville de Montréal $^{3}$ a choisi une autre voie, depuis 1994. Sans s'intéresser directement à la sortie de rue des pairs qui le composent, cette stratégie d'intervention permet de donner un rôle d'intervention à des jeunes ayant vécu dans la rue, dans le cadre d'un dispositif d'action concertée visant la prévention de la transmission du VIH et des autres ITSS et la réduction des méfaits de la toxicomanie et de l'utilisation des drogues injectables, chez les jeunes qui fréquentent le centre-ville.

Le Collectif d'intervention par les pairs a été créé à l'origine par des intervenants de la Clinique des Jeunes St-Denis, dans une période où le nombre de jeunes de la rue semblait en croissance à Montréal et où les interventions habituelles, tant en itinérance qu'en jeunesse, ne parvenaient pas à rejoindre cette catégorie de jeunes (Direction de la Santé publique, 1998). Il vise à associer des pairs-aidants avec différents

de santé et des services sociaux, mais aussi entre le réseau des services et la rue, en dépassant les rapports habituels de normalisation que les jeunes de la rue ont connus, dont le but est de les sortir à tout prix de la rue. Pour une analyse détaillée des résultats de cette recherche, voir Bellot et al., 2006.

2. Cette recherche, portant sur l'expérience de la sortie de rue et dirigée par Céline Bellot, a été financée par le CRSH. Elle visait à appréhender le processus de sortie de rue, en s'intéressant à comprendre les opportunités et les obstacles qui facilitent ou entravent le travail identitaire et le repositionnement social de ces jeunes entre la marginalité et la conventionalité. Différents «échantillons» ont été mis à contribution pour rencontrer des jeunes dans diverses ressources ayant un mandat d'hébergement ou de logement supervisé, un mandat thérapeutique ou encore d'insertion sociale et professionnelle. Des jeunes ont aussi été rencontrés en dehors des structures de ressources et finalement, à l'occasion du $10^{\mathrm{e}}$ anniversaire du Collectif et des retrouvailles organisées dans ce cadre, nous avons rencontré la plupart des jeunes ayant été pairs ou participant encore à cette intervention. Dans toutes ces rencontres, nous voulions comprendre comment ils avaient vécu la rue, leur engagement dans le Collectif et pour ceux qui n'y étaient plus, ce qu'ils étaient devenus depuis. Ce dernier «échantillon» a fait l'objet d'une analyse spécifique dans le cadre d'un mémoire de maîtrise à l'Université de Montréal (voir Greissler, 2007). Ce sont les données de trajectoires concernant les pairs et les anciens pairs qui seront mises à contribution dans cet article.

3. Voir Bellot et al. (2006) pour la description détaillée du Collectif. 
organismes partenaires institutionnels et communautaires. Les pairsaidants travaillent 28 heures par semaine et sont payés $10 \$ / \mathrm{h}$, soit un taux horaire légèrement supérieur au salaire minimum. Ils reçoivent une formation tout au long de leur engagement dans le Collectif. Dans les organismes partenaires, ils sont jumelés à un intervenant-superviseur, qui leur permet de soutenir le déploiement d'interventions individuelles. La façon d'intervenir des pairs-aidants complète celles des autres intervenants. Ils réussissent généralement à rejoindre les jeunes dans leur milieu de vie, là où habituellement les autres intervenants ont peu d'accès. Les pairs-aidants réalisent des interventions sur le plan individuel, par l'écoute, le soutien et l'accompagnement et sur le plan collectif par l'organisation d'évènements spéciaux, comme le Festival d'expression de la rue ou bien le Show Punk Hépatite C. Il n'y a pas de durée d'engagement dans le Collectif des pairs. Certains pairs-aidants restent six mois, d'autres y demeurent deux ans, mais la durée moyenne d'engagement dans le Collectif est un peu plus d'un an. La plupart des pairs-aidants vont par la suite trouver un emploi dans l'intervention sociale, certains vont retourner aux études, fonder une famille, d'autres vont s'engager dans des démarches artistiques et finalement quelquesuns retourneront dans la rue.

Après avoir présenté les différentes manières d'envisager la sortie de rue et les principales caractéristiques et modalités de l'intervention par les pairs, notamment celles développées au sein du Collectif, nous montrerons comment ce dispositif permet aux pairs-aidants d'endosser un nouveau rôle, d'acquérir une expérience et d'être reconnus, en inscrivant nos analyses dans une perspective de reconnaissance de la construction identitaire de l'individu, indépendamment de son espace de socialisation et du regard normatif posé sur celui-ci.

\section{Un bref survol des conceptualisations de la «sortie » de rue}

Nombre d'écrits sur les jeunes de la rue portent sur les causes et les conséquences de l'expérience de rue et s'intéressent à la nature et au sens de leurs activités quotidiennes, notamment en ce qui a trait à l'idée de leur socialisation. Les nombreuses descriptions des parcours et les différentes compréhensions de leurs «trips» focalisent souvent sur le même instant, soit l'expérience de rue proprement dite ou sur les raisons de l'arrivée dans la rue. Cette expérience est alors le plus souvent tra- 
duite en termes de danger, de risques, le jeune devenant alors tantôt victime, tantôt délinquant. La rue est perçue alors comme diabolique car soustraite à l'influence de tout adulte (parents, autorité), et laissant donc toute la place à l'influence des pairs, considérée comme néfaste. Ces études témoignent de cette construction de l'impérieuse nécessité de la sortie de la rue (Bellot, 2001; Colombo, 2008). D'autres études, moins nombreuses, tentent d'appréhender ces jeunes comme des acteurs et de montrer comment l'expérience de la rue peut être un espace d'apprentissage et de construction identitaire. La sortie est alors décrite comme un désir de changement du jeune lui-même, désir qui va s'incarner dans un processus où le jeune expérimente de nouvelles voies d'ancrage dans son rapport à la société.

\section{La rue vue comme un danger et la sortie imposée}

De nombreux travaux présentent des éléments portant, par exemple, sur la dangerosité de l'expérience de rue et les risques qui en découlent, comme la marginalité, la précarité, la délinquance et aussi la mort. Ainsi, Hagan et McCarthy (1998) dès les premières pages de leur étude plantent le décor: l'expérience de la rue est une expérience extrême de survie. Dès lors, le passage dans des activités délinquantes s'appuie à la fois sur cet espace hostile et sur le rôle des pairs déviants que les jeunes y côtoient. Côté (1988), l'une des premières auteures à s'être intéressée aux jeunes de la rue au Québec, va problématiser son étude autour des causes et des conséquences de ce «processus» de quête de soi, dans un milieu plutôt hostile, espace de déviance. Elle considère ainsi la rue comme une impasse sociale et un risque d'exclusion sociale. Pattegay (2003) explique comment, dans ces études, la rue est un lieu «d'errance». Chobeaux (1996) parle quant à lui de «nomades du vide» puisque les jeunes de la rue ne sont pas soumis aux mécanismes habituels de socialisation qui permettent aux adultes et notamment aux parents de soutenir la construction identitaire et le développement de leurs enfants. Les jeunes de la rue tournent en rond ou foncent dans un mur tant leurs perspectives d'avenir semblent cloisonnées (Chobeaux, 1996; Chazy, 1999; Tremintin, 1999). Finalement, les études d'Élise Roy, qui suivent une cohorte de jeunes de la rue à Montréal, montrent comment les jeunes de la rue présentent un taux de mortalité neuf fois supérieur à d'autres jeunes de même catégorie d'âge (Roy et al., 1998, 2000). 
Ces images de perte, de risque et de dangerosité affluent pour soutenir cette conceptualisation "diabolique» de la rue et alimentent l'impérieuse nécessité de sortir les jeunes de cet espace. Dans ce sens, les schémas de la «sortie» sont prédéterminés. Il s'agit, par exemple, d'après Caputo et al. (1997), de retrouver le droit chemin «acceptable» et donc de rompre avec le style de vie de rue. L'objectif de l'intervention sociale est alors, critiquent certains auteurs, la «démarginalisation» des jeunes de la rue, soit la «normalisation» de leurs conduites (Bellot, 2001; Colombo, 2001; Lucchini, 2001; Rivard, 2004). Pourtant, d'autres études contestent cette vision négative et normalisée de l'expérience de rue.

\section{Des études sur la production identitaire marginalisée}

Certains points de vue permettent, en effet, d'envisager autrement ce passage des jeunes dans la rue. Ainsi, Parazelli (1998, 2002, 2003) défend l'idée selon laquelle la rue n'est pas un espace détaché de la société ou encore vide de sens, parce qu'il permet justement aux jeunes de vivre une socialisation dans des "formes concrètes de relations sociales» (Parazelli, 2002: 137). La rue devient un espace de «socialisation marginalisée», qui permet aux jeunes de s'ancrer dans «le social», par ses marges. S'appuyant sur son concept «géosocial», Parazelli montre que les jeunes s'inventent de nouveaux repères pour la réalisation et l'expression de soi, dans le contexte actuel d'incertitudes. Ainsi, au cœur d'un ensemble de dimensions géographiques et sociales, la rue est perçue comme un «espace transitionnel».

Pour Colombo (2001), la «sortie» constitue un passage, selon des rites anthropologiques. Pour illustrer son propos, elle décrit l'enfermement du jeune dans la rue ou plutôt dans une illusion de liberté qui s'essouffle. Face à une pression sociale et en regard des difficultés vécues avant et dans la rue, le jeune remettrait alors en question son mode de vie de rue et rechercherait un style de vie différent. Selon un «processus » à travers lequel l'expérience de rue se modifie ou disparaît, le sujet comprend qu'il peut faire face à son avenir et en devenir l'acteur. Il peut alors se projeter et effectuer son passage de l'enfance à la vie adulte, de façon singulière et personnelle. L'auteure, tout comme Sheriff (1999), définit cette période de transition identitaire comme une fin des expériences de rue, soit une «sortie» de rue. C'est en fait, une période de réaménagement du mode de vie, selon des normes plus 
traditionnelles que sont l'emploi, le logement, la non-consommation de drogues et enfin - et peut-être surtout - la non-fréquentation de la rue. Bergier (2000) parle alors d'affranchissement de la position marginale. L'intervention sociale serait, dans ces circonstances, pensée comme une modalité d'accompagnement dans cette quête de conformité des jeunes.

Si cette perspective se rapproche de la nôtre, dans la mesure où elle permet de montrer comment l'expérience de rue peut être appréhendée comme un processus de socialisation participant à la construction de l'identité des jeunes, elle ne parvient pas, dans les analyses de la sortie de rue, à considérer l'expérience de rue comme un élément à intégrer. $A$ contrario, cette expérience est présentée comme une expérience à effacer, en raison de la quête de conformité qu'auraient les jeunes (Bellot, 2001). En ce sens, il s'agit pour nous de se positionner autrement que par l'impérieuse nécessité de sortir les jeunes de cette expérience de rue et de démontrer comment tant la société que les jeunes eux-mêmes peuvent gagner à intégrer cette expérience de rue en lui reconnaissant une valeur certaine.

\section{Penser la sortie de rue comme un processus d'intégration biographique de l'expérience de la rue}

Ancrer nos analyses de la sortie de rue dans un paradoxe, où il s'agit d'appréhender comment les jeunes en situation de rue tentent d'intégrer cette expérience dans leur récit de vie, constitue pour nous une manière de saisir la globalité des modes de socialisation de ces jeunes, en soutenant que la rue, loin d'être un espace de transition identitaire, peut être considérée comme un espace de révélation, d'adhésion à des valeurs, de diverses formes de relations et de normes (Bellot, 2001). Ainsi, nous définissons la sortie de rue comme un processus d'intégration biographique de l'expérience de la rue, qui va d'un affranchissement complet à une affirmation absolue de cette expérience. Dans ces termes, il s'agit de s'intéresser au travail biographique que réalisent ces jeunes, à travers l'examen de leur vécu, la compréhension et la structuration de leurs expériences et de leurs actions qui leur permettent de devenir quelqu'un, c'est-à-dire de bâtir une biographie, entendue ici comme un récit de soi (Delory-Momberger, 2004). En ce sens, le travail de production de soi des jeunes en situation de rue s'apparente à celui de n'importe quel individu dans nos sociétés modernes. Il exige de l'individu d'intégrer 
et d'interpréter, à travers un cadre de structuration et de signification de l'expérience, une histoire qui le définit (Delory-Momberger, 2004).

Or, l'analyse de ce travail biographique réalisé par des jeunes en situation de rue permet de révéler les tensions paradoxales auxquelles sont soumis les individus dans nos sociétés contemporaines. En effet, la plupart des perspectives théoriques sur l'identité contemporaine tentent de montrer comment les formes de socialisation font une part croissante aux processus d'individualisation et de subjectivation (Ehrenberg, 1996; Lahire, 1998; Bajoit, 2000, 2003; Martucelli, 2005). Les individus sont sommés de se bricoler une identité, de construire leur biographie, qui leur permet de faire preuve d'initiative, d'autonomie, de créativité par rapport aux modes traditionnels de socialisation. Somme toute, l'individu-acteur est devenu le propre pilote de son travail biographique, en traversant un ensemble d'opportunités et de contraintes, avec lesquelles il tente de construire sa propre trajectoire (Dubet, 1995).

Dans cette perspective et s'appuyant sur les théories de la structuration, qui permettent de lire la marge de manœuvre de l'acteur à l'égard du système dans sa production identitaire, cet article se veut également une occasion de soutenir la compréhension de travail biographique. C'est donc grâce à l'analyse de ce paradoxe - système vs production identitaire - que nous avons choisi de lire le travail biographique des jeunes qui vivent dans la rue à partir de l'étude de l'intégration de cette expérience dans leur histoire, postulant que les conditions de ce possible seraient révélatrices des tensions paradoxales auxquelles sont soumis les jeunes en situation de rue: pris d'une part entre l'opportunité d'une émancipation à travers des expériences non ordinaires de socialisation - respectant en cela l'injonction de la construction identitaire autonome de nos sociétés contemporaines - et l'exigence de la normalisation qui impose aux jeunes l'occultation de cette expérience de leur biographie. Ainsi, à travers les récits de ces jeunes, nous avons colligé ce qu'ils retiennent ou non de leur inclusion dans le monde social de la rue en tant qu'expérience de socialisation singulière (Dubet, 1995). Avant de rendre compte du point de vue des jeunes sur leur biographie, il est nécessaire de définir et de caractériser l'intervention par les pairs de manière générale et celle du Collectif d'intervention par les pairs plus spécifiquement. 


\section{L'intervention par les pairs: une manière de repenser les relations d'intervention}

Les stratégies d'intervention par les pairs sont nombreuses et se sont multipliées au cours des dernières décennies, adoptant des approches préventives ou curatives (Milburn, 1995; Sciacca et al., 1996; Backett et al., 2000; Fontaine et al., 2006; Proulx et al., 2006; Bellot et al., 2007). Malgré leur pluralité, elles ont toutes en commun de positionner un individu ou un groupe d'individus dans un rôle différent de celui qu'il entretient généralement avec des individus qui lui ressemblent. En effet, l'intervention par les pairs exige la proximité dans les relations et une forme de reconnaissance afin de jouer un rôle associé à l'intervention (Bellot et al., 2007). Que le registre de l'action soit celui de l'influence, de l'entraide ou de la médiation, les pairs se positionnent dans des relations avec leurs pairs à titre de modèles, s'appropriant des stratégies qui visent à établir autrement les rapports d'intervention.

Le dispositif d'intervention du Collectif d'intervention par les pairs rassemble autour de lui des pairs, des intervenants superviseurs, des partenaires et une coordonnatrice. Les pairs sont au cœur du Collectif, dans la mesure où ils assument la concrétisation de l'intervention tant sur le plan individuel que collectif, dans leur alliance partenariale avec les ressources d'accueil, dans leur lien avec les jeunes, les activités spéciales qu'ils organisent. De plus, ils participent à la définition des orientations du projet par leur participation réelle et volontaire à chacun des processus décisionnels instaurés dans le cadre du projet, incluant le comité de coordination. Placés dans ces différentes instances, ils incarnent le rapprochement entre le monde de la rue et le monde de l'intervention socio-sanitaire. Pour devenir pair, il faut avoir connu la rue, avoir pris du recul par rapport à ce mode de vie depuis un an, notamment en termes de consommation de drogues injectables, avoir un intérêt pour le rôle d'aidant ainsi que pour la prévention des ITSS ${ }^{4}$.

4. Ces critères ont été créés et raffinés au fil des années. Moins exigeant, notamment en termes de contrôle de la consommation à l'origine, le Collectif a souhaité mettre cette exigence de côté afin de faciliter la stabilité du groupe de pairs. Toutefois, il importe que les jeunes recrutés soient encore en lien avec la rue. Le recrutement exige donc systématiquement de travailler à maintenir un équilibre entre capacités des candidats à réaliser de l'intervention et proximité et intimité avec les jeunes visés, soit les jeunes de la rue. En outre, à chaque recrutement d'un nouveau pair, le Collectif vise à maintenir une diversité dans le groupe afin de représenter la pluralité de la rue, en termes de genre et de souscultures. 
Leur nombre varie autour de six et ils réalisent autant d'alliances avec des organismes communautaires ou institutionnels, au sein desquels ils intégreront les équipes de travail, pour environ la moitié de leur temps de travail et apporteront une perspective supplémentaire à la prévention. L'autre moitié de temps de travail concerne l'ensemble du groupe de pairs et la coordonnatrice et vise à dynamiser le dispositif partenarial, à structurer des activités spéciales collectives dont la plus importante demeure le Festival d'expression de la rue s. $^{5}$

Les partenaires, pour leur part, participent au Collectif en permettant à chaque pair une forme de jumelage avec leur organisme pour bâtir une intervention alternative auprès des jeunes de la rue qui le fréquentent. Très diversifiés, on retrouve des organismes communautaires en même temps que des instances du réseau public. Autour de la table des partenaires: un programme d'échange de seringues, un centre de jour, des ressources d'hébergement, une ressource d'intervention mobile, une équipe santé jeune de la rue du réseau public, une équipe jeunesse d'un centre de réadaptation en matière de toxicomanie, une ressource de défense des droits des travailleuses du sexe. Ces organismes représentent également la diversité de la rue, par le type de services et d'approches distincts qu'ils offrent: de l'hébergement à l'échange de seringues; du travail de rue à la réadaptation.

Dans chaque ressource partenaire d'accueil, un intervenant joue un rôle particulier de supervision du travail du pair, en vue de soutenir sa formation à l'intervention, mais aussi d'intégrer de plus en plus le pair à l'organisme. En outre, les intervenants superviseurs participent au bilan annuel du projet d'intervention par les pairs. Leur rôle est crucial pour le pair et pour le projet dans son ensemble, car c'est dans le cadre de ces relations privilégiées que se définit la reconnaissance du pair dans la ressource, son intégration positive à ses nouvelles fonctions dans le milieu plus général, tout comme sa formation.

Tout le Collectif est sous la supervision d'une coordonnatrice qui veille, au quotidien, au fonctionnement du projet et au maintien du difficile équilibre entre le monde de la rue et le monde de l'intervention socio-sanitaire. Elle est accompagnée dans sa tâche par un comité de coordination qui incarne le cadre partenarial du projet. Ce comité est

5. Chaque année, durant trois jours et ce depuis bientôt 10 années, le Collectif organise ce festival dans un parc du centre-ville et dans le cadre duquel des jeunes présentent différentes prestations (musique, cirque, documentaires, films, radio, poésie, graffiti). 
composé, en plus de la coordonnatrice, de représentants des organismes communautaires, des organismes publics, des bailleurs de fonds, de personnes engagées depuis le début du projet et enfin, des pairs. Le rôle du comité de coordination est de définir les orientations du projet, veiller à son bon fonctionnement, soutenir le travail de collaboration et encourager la définition d'objectifs communs d'intervention. Il est, en somme, l'espace collectif décisionnel, là où s'harmonisent les différents points de vue des acteurs, le lieu de la gouverne collective du projet, bref, son cadre partenarial. Ce comité se réunit quatre fois par année. Au fil du temps, il a permis de consolider le projet dans sa structure partenariale, mais aussi de débattre des fondements du projet, de ses valeurs, de sa mission et de ses mandats, et d'en assurer la transmission auprès des nouveaux partenaires.

L'examen d'un tel dispositif prend tout son sens lorsqu'il est mis en relation avec notre questionnement sur l'intégration biographique de l'expérience de la rue dans la trajectoire des jeunes. En effet, dans la mesure où même s'il est construit dans une logique de prévention et qu'il «cible» les jeunes de la rue, le Collectif d'intervention par les pairs constitue, pour les pairs, un véritable espace d'opportunités de reconnaissance de leur expérience de rue. Nous le montrerons après avoir présenté la méthodologie des deux recherches mises à contribution.

\section{Démarche méthodologique: une analyse croisée de deux recherches}

Comme on l'a vu plus tôt, les résultats de la présente étude s'appuient sur l'analyse croisée de deux recherches conduites autour du Collectif d'intervention par les pairs qui sont brièvement présentées ici.

\section{La recherche évaluative du Collectif d'intervention par les pairs}

Le cadre méthodologique de la recherche évaluative concernant le Collectif d'intervention par les pairs du centre-ville de Montréal s'inscrit dans une posture épistémologique à la fois participative et formative, à partir d'une démarche de recherche qualitative, qui renvoie à la procédure proposée par Yin (1994) pour l'analyse de cas multiples. Ce choix méthodologique s'explique par le fait que chaque pair est associé à un organisme partenaire et qu'ainsi, chacun des couples jumelés est 
devenu un cas. L'équipe a eu recours, en outre, à une stratégie méthodologique double: verticale, par l'étude de cas, pour appréhender en profondeur le travail d'un pair dans sa ressource, et transversale, pour saisir davantage la globalité du projet et sa dimension collective. Au moment de l'évaluation, six pairs participent au projet d'intervention et sont rattachés à six organismes différents. Six études de cas ont donc été réalisées.

Utilisant l'observation participante comme socle de la méthodologie évaluative, la démarche a été complétée par l'analyse de données déjà intégrées dans les activités d'intervention des pairs: bilan des jeunes contactés; rapport d'activités de groupe; journal de bord; bilan personnel de fin de projet. À cette analyse s'ajoute l'examen des données générées par l'équipe de recherche: entrevues de groupe; entrevues individuelles auprès de jeunes de la rue, des pairs et de divers acteurs associés au Collectif; et finalement, focus groups avec les organismes partenaires.

\section{La recherche sur les sorties de rue}

Inspirée de l'approche théorique sur la biographie, présentée antérieurement, l'étude de Greissler (2007) repose sur les récits de vie de pairs et d'ex-pairs aidants. Cette méthodologie relève des principes avancés par Bertaux (1997) qui a (ré)introduit le procédé de «récit de vie» en sociologie. De cette manière, nous avons pu resituer les actions des pairs et des ex-pairs dans leur contexte concret (social et historique). Dix-huit personnes ont participé à cette étude, entre novembre 2004 et mars 2006. L'âge moyen des pairs et des ex-pairs rencontrés est de 26,5 ans. Ils ont tous pris un recul face à leurs expériences de socialisation marginalisée de 5 à 6 ans en moyenne. Tous également ont été dans le Collectif des pairs aidants à des époques différentes, certains en faisaient encore partie au moment des entrevues. Cette diversité des situations apporte une variété de témoignages et révèle des expériences aussi riches qu'hétérogènes.

\section{Le travail de recomposition identitaire au sein du Collectif des pairs}

Comme nous l'avons présenté, le Collectif des pairs fait figure de relais entre deux mondes, enjoignant du même coup à ses participants de se 
positionner en relais à leur tour. En devenant pairs, ces jeunes qui ont connu la rue s'inscrivent dans un processus d'expérimentation et d'exploration dans lequel les transformations des rôles et des positions qu'ils occupent dans le monde de la rue, comme dans le monde de l'intervention socio-sanitaire, de même que les processus de changements biographiques qu'ils vivent, contribuent à leur construction identitaire. Certes, ce travail de construction dépasse le cadre relatif de leur participation au Collectif des pairs, mais il en fait partie aussi. Ainsi, ces jeunes se construisent par l'intermédiaire et au-delà du Collectif. Pourtant, un examen attentif de leur expérience dans le Collectif des pairs fait voir comment ce dernier permet l'intégration biographique de l'expérience de rue, en reconnaissant le potentiel des jeunes et en leur offrant une opportunité de travail d'intervention. En ce sens, l'intégration biographique de l'expérience de rue des pairs-aidants rend compte du travail identitaire qu'ils réalisent, des expériences qu'ils acquièrent dans ce collectif et de la nécessité de voir reconnaître cette dimension de l'intégration biographique.

\section{Devenir pair-aidant, c'est devenir un modèle}

L'intégration d'un jeune comme pair-aidant dans le cadre du Collectif comporte de multiples facettes et de nombreuses exigences pour le jeune. Outre le fait de se retrouver dans un rapport salarié pour 21 à 28 heures, les jeunes qui deviennent pairs doivent tout d'abord intégrer ce rôle d'équilibre entre les deux mondes présentés plus tôt. Ainsi, dans les premiers temps, le pair doit apprivoiser cette tension distance/proximité avec le monde de la rue, qui va favoriser son insertion comme pair-aidant dans le Collectif. Pour cette raison, le Collectif assure de nombreuses formations aux jeunes, en vue de les soutenir dans la familiarisation avec ce nouveau rôle. Les jeunes qui deviennent pairsaidants ont généralement des aptitudes pour la relation d'aide qui leur paraît naturelle et qu'ils semblent avoir toujours eue. On verra souvent chez les pairs un surinvestissement du temps d'intervention, d'autant plus qu'entre les périodes où ils sont dans leur rôle de pair-aidant et celles où ils sont simplement des amis par exemple, les frontières sont poreuses et ce, en raison de la nature même du projet:

Durant le retour, entre deux arrêts d'autobus, A., la superviseure, demande à J., la nouvelle pair-aidante, ce que son rôle de pair-aidante implique sur le plan des limites. J. répond qu'elle est plus flexible qu'une intervenante, 
qu'elle affiche moins de distance, qu'elle peut parler de son passé, de son expérience personnelle. Elle dit aussi pouvoir les voir [les jeunes de la rue] plus souvent et plus longtemps. A. met alors en garde J.: « Trop s'impliquer peut amener au burnout.» (Extrait du journal de bord, observation du 22 janvier 2003)

La question de la proximité s'exprime aussi fréquemment dans le rôle de transmission d'informations que les pairs-aidants jouent. On sait que les dispositifs d'intervention par les pairs visent d'abord et avant tout le transfert d'informations par des individus qui sont crédibles et entendus, dans la mesure où ils partagent un univers commun. Au cour de la tension distance/proximité s'incarnent donc pour les pairs des rapports d'influence. Une tension qui impose d'atténuer les frontières entre les rapports d'intervention et les rapports d'intimité:

Moi j'ai l'hépatite $\mathrm{C}$ et j'en parle avec mes amis et mon entourage quand je suis à l'aise de le faire et souvent ils me posent beaucoup de questions et je leur donne beaucoup d'informations. Et pis la formation m'a aidé à compléter mes infos. Mais tu sais, du coup je peux leur dire: «Tu sais moi quand je prends trop de bière, le lendemain c'est vraiment lendemain de veille et c'est pas drôle car ce que j'attaque c'est vraiment mon foie», et pis j'suis capable d'expliquer comment l'alcool alimente le virus. (Pair-aidant, B.)

Lorsque les pairs-aidants sont interrogés sur le rôle qu'ils exercent, ils vont aussi s'attarder à faire une distinction avec le rôle d'intervenant traditionnel:

J'avais de la misère à faire mon travail quand je me disais que je devais faire mon mandat, la prévention VHC, comme les autres intervenants. Au début, je me disais que je devais parler de condoms au lieu d'écouter le jeune raconter sa vie, parce que je devais faire de la prévention. Mais j'ai vite compris, ça marche pas. Ça sert à rien de donner des condoms si tu sais rien sur le jeune. Notre job c'est pas un mandat. C'est bien davantage une mission d'aide. On est là pour servir de modèle. Car t'sais dans la rue, il n'y a pas beaucoup de modèles positifs. Moi je veux être un modèle pis que les jeunes viennent me voir en étant eux-mêmes. Pis t'sais même pour moi c'est de montrer qui je suis vraiment, dire par quoi je suis passé, mais qui je suis maintenant. Mais c'est pas pour qu'eux y fassent pareil. C'est juste pour donner une petite voix qui leur dit, c'est important de te connâ̂tre pis de faire des choses pour toi. (Pair-aidant, S.)

Au-delà des fondements de l'intervention par les pairs qui visent les registres de l'influence et de l'éducation, dans un contexte où le message devient crédible puisque le messager l'est, ce qui semble être davantage 
présent pour les pairs-aidants, c'est l'idée du modèle. Les contours de ce modèle, dans les mots des pairs-aidants, ne représentent pas une manière de définir un parcours que les autres devraient suivre. Il s'agit, bien au contraire, de montrer qu'un bricolage identitaire est possible et nécessaire pour devenir un individu dans la société, participant de la sorte au processus de subjectivation et d'individuation qui permet à la personne de se bâtir singulièrement. L'idée du modèle est constante dans les mots des pairs. Voilà ce qu'ils partageaient dans le cadre d'un focus group à notre question: «Qu'est-ce qu'un pair-aidant?» :

Un ancien jeune de la rue qui fait de l'intervention avec d'autres jeunes de la rue; plus de liberté qu'un intervenant formel. (Pair-aidant, S.)

Être une référence, un modèle de persévérance pour les jeunes. Leur montrer qu'il y a quelque chose au bout de la rue et faire le lien avec les autres intervenants sur la rue et les autres du motorisé, mais faire le lien avec d'autres organismes aussi. (Pair-aidant, J.)

C'est être un modèle positif. C'est de montrer aux jeunes de la rue et adultes que peu importe ton passé, que tu peux réussir à changer de mode de vie, que tu peux faire ce que tu aimes, que tout est possible. C'est de faire de l'intervention et d'aider mes pairs. (Pair-aidant, B.).

Établir un réseau autour des jeunes pour qu'ils aient de l'aide sans que j'aie besoin d'être là. Anciens jeunes de la rue qui se récupèrent pour aider d'autres personnes en ayant des formations, visites. (Pair-aidant, V.)

Intervenant, mais venant du milieu de la rue. (Pair-aidant, R.)

À travers ces quelques mots et la définition de ce qu'ils font, il est possible de voir comment ils valorisent leur trajectoire alors qu'ils témoignent du chemin qu'ils ont parcouru. L'idée d'un modèle positif tend, dès lors, à montrer comment ils sont parvenus à intégrer leur expérience de rue. En ce sens, le travail d'intégration biographique, révélé à la fois par le statut et le rôle de pair-aidant, devient ainsi dans leur discours l'occasion de témoigner de ce travail, en faisant état des processus d'individuation et de subjectivation qu'ils vivent. Faire le récit de leur intégration biographique de l'expérience de rue et se positionner comme modèle, équivaut, pour ces jeunes, à dire la «normalité» de leur marginalité et, du même coup, à soutenir le parti pris d'une revendication identitaire de différenciation qui s'exprime à travers un positionnement défini comme marginal.

Cette tension entre une marginalité "négociée», «revendiquée», «assumée», selon les termes des jeunes rencontrés, s'accompagne aussi 
d'un besoin identitaire de se situer sur le plan des valeurs. L'ancrage à la marge constitue, pour ces jeunes, une manière de définir et de réaliser leur opposition aux valeurs de la société contemporaine. Ils font figure parfois davantage de pionniers, de précurseurs, d'avant-gardistes ou d'alternatifs, et ne collent plus à ces images de déviants et de délinquants que le regard normatif extérieur persiste à poser sur eux.

\section{«Être pair-aidant, c'est acquérir une expérience sans nier qui nous sommes»}

Très souvent, la sortie de rue rime avec une rupture brutale du milieu dans lequel les jeunes ont évolué pendant plusieurs années (Greissler, 2007). L'intégration dans le Collectif des pairs-aidants constitue une opportunité particulière dans la mesure où elle va permettre de se maintenir dans la rue tout en y jouant un nouveau rôle ou un rôle qui s'officialise. Ainsi, d'un côté, les pairs-aidants ont un rapport d'employé, avec un salaire et des obligations de travail qui s'y rattachent et, de l'autre côté, ils continuent de fréquenter le milieu qui est le leur. Le fait que le Collectif n'ait aucun objectif portant sur le cheminement des pairs-aidants - l'intérêt étant centré sur les jeunes de la rue - impose à ces pairs un rapport complet d'employé qui les distingue par exemple de jeunes qui sont intégrés dans des programmes d'insertion socioprofessionnelle où la cible est le jeune participant lui-même:

La pair jumelée à X. est présente depuis maintenant plus de deux ans. Elle effectue diverses actions auprès des jeunes, comme le montrent ses grilles d'intervention qu'elle remplit régulièrement: prises de contact, écoute active, support, prévention ITS-VIH-VHC, etc. Le simple fait de remettre les effets personnels aux jeunes, d'être à l'accueil, est souvent un prétexte de prise de contact avec les jeunes. De plus, B. a, tout au long de l'année, intensifié sa participation aux réunions informelles tenues par les intervenants. Ces rencontres quotidiennes constituent un lieu d'échanges où $\mathrm{B}$. apporte une contribution en termes d'informations concernant les jeunes moins connus des intervenants. De plus, B. note dans les dossiers de certains jeunes les informations qu'elle juge pertinentes. (Entrevue, intervenant superviseur, J.)

À travers cet extrait, il est possible de constater le positionnement du pair-aidant dans sa ressource d'accueil en tant qu'intervenant, singulier il est vrai, mais néanmoins intervenant tout de même, dans la mesure où il est possible de décrire ce qu'il fait, comment il en rend compte et 
comment son travail se lie à celui des autres intervenants. Ainsi donc, si le rôle de pair-aidant s'apparente à un réel emploi d'intervenant, il se construit cependant au fur et à mesure de l'évolution du pair dans le projet et dans sa ressource d'accueil. Pour la plupart des anciens pairsaidants que nous avons rencontrés, l'expérience dans le Collectif a été déterminante en ce sens qu'elle a permis l'acquisition d'un savoir-faire professionnel qu'ils ont pu utiliser ultérieurement. Par exemple, plus de la moitié des pairs-aidants rencontrés sont devenus intervenants sociaux dans différents milieux et organismes. Le passage par le Collectif apparaît clairement comme un tremplin qui donne accès au marché du travail traditionnel, soit au passage entre les deux mondes que nous avons décrits plus tôt.

En somme, cette expérience n'impose pas aux pairs de renier leur passage dans la rue, ce qui pour la plupart constitue ou a constitué une clé d'abord pour susciter leur intérêt au projet, ensuite, pour soutenir le travail de compromis identitaire qui leur incombe entre leurs revendications de la marginalité et la vie en société. Rarement promu, c'est en effet un moyen de rester proche de ses valeurs que de jumeler, en tant que pair et aussi en tant que personne, des éléments de conformité et de marginalité. Soucieux de demeurer authentiques par rapport à leurs valeurs, leurs expériences et leurs relations sociales, tout en cherchant à vivre autrement que dans la rue, les pairs qui témoignent de cette forme d'influence montrent comment le Collectif les a aidés à progresser dans la tension paradoxale qu'ils vivaient entre la marge et la norme. Ainsi, le Collectif leur a permis de clarifier l'expression de ce qu'ils sont, en leur fournissant un espace de reconnaissance où ils pouvaient être distincts tout en étant reconnus, en occupant un rôle social accepté, voire valorisé. À travers son expérience de pair-aidant, O. a réfléchi sur la place qu'il occupe dans la société et les moyens d'exprimer sa différence:

C'est la première opportunité de faire quelque chose qui peut te plaire... comme nous [dans un de ses projets], on l'a envisagé dans cette logique-là [...], c'est-à-dire que travailler peut devenir un plaisir [...] c'est pas comme un fardeau [...] l'idée de travailler c'est comme une concession sur la liberté, une concession sur la vie... et puis les pairs-aidants, pour beaucoup de gens, c'est comme cette transition-là, d'autant plus qu'il y avait beaucoup de tolérance dans le système des pairs-aidants, ce qui est bien et qui était comme nécessaire pour que les gens en profitent pour qu'ils réalisent ce qu'ils sont avec le temps [...] je pense que ça m'a permis de concrétiser des choses ce projet-là... je pense que ça a été hyper formateur [...] d'ailleurs 
je l'ai pas dit durant toute l'entrevue, je pense que ça a été un accomplissement pas tant personnel que collectif dans une société comme dans une collectivité [...] pour moi ça a concrétisé cette question-là [...] expérimenter cette espèce de philosophie-là que j'avais en tête, qui est fondamentale [...]. (Entrevue, ancien pair, O.)

Pour d'autres pairs-aidants, notamment de sexe féminin, l'influence du dispositif s'exprime dans le désir de prendre soin de soi. Elles racontent alors comment leur parcours de rue a été marqué par la consommation de drogues, consommation dont elles souhaitent maintenant se protéger même si le désir de rester en contact avec la rue est fort ${ }^{6}$. Dans ces situations, le Collectif permet de créer une dissociation entre le monde de la rue et le monde de la drogue, en proposant un cadre d'interactions orienté vers la prévention et le souci de soi. Enfin acceptés et reconnus, ces pairs vont utiliser le Collectif comme un espace de construction de la confiance en eux. Dans ce contexte, le Collectif constitue souvent un trait d'union, un cadre professionnel sécuritaire, qui éloigne le jeune pair $\mathrm{du}$ monde de la consommation de drogues en lui permettant, tant sur le plan individuel que sur le plan de l'intervention, de mettre de l'avant son aspiration à mieux prendre soin de lui. Le Collectif des pairs lui permet en quelque sorte, une réappropriation du monde de la rue où les comportements destructeurs font place à des attitudes de prévention:

L'exemple typique de ce rapport de protection de soi concerne une paireaidante que nous avons observée durant plusieurs mois dans le cadre de son intervention. Ayant connu une consommation par drogues injectables importante, elle appréhende son rôle de pair-aidante en mettant de l'avant la distribution constante de matériel d'injection. Parmi les pairs observés, c'est celle qui mettra le plus d'emphase sur ce rôle de distribution. Devenue «madame matériel» dans sa ressource, elle développe l'ensemble de ses relations d'intervention autour de l'importance d'adopter un cadre sécuritaire de consommation. Avec elle, la distribution se fait sans en avoir l'air, sans que cet échange de matériel soit au cœur du rapport social. Par contre, elle conclut l'ensemble de ses échanges par un «prends soin de toi». D'un point de vue personnel, son rôle de paire est devenu pour elle un espace

6. La question de la consommation de drogues ne concerne pas seulement les jeunes filles qui vivent dans la rue. Cependant, dans les deux recherches mises à contribution ici, ce sont elles qui l'ont exprimé le plus souvent, dans un rapport de distanciation au monde de la rue, en raison des insécurités qu'elles y vivaient. Passer à autre chose, c'est le plus souvent pour ces jeunes filles, se positionner dans un rapport de protection vis-à-vis de la société. Certaines d'entre elles ont peur d'elles-mêmes et de la rue et utilisent le Collectif tel un rempart contre le monde de la drogue dans la rue (Greissler, 2007). 
thérapeutique. Chaque jour passé dans le Collectif est un jour sans consommation. Des circonstances biographiques l'ont conduite quelque temps après notre évaluation à une rechute dans la consommation. Lorsque nous avons cherché à la rencontrer ultérieurement pour la réalisation de la recherche, elle était partie en région éloignée (700 km de Montréal), sa région d'origine. Elle nous dira alors au téléphone ${ }^{7}$ que malgré une expérience positive dans le Collectif des pairs, Montréal était une ville trop dangereuse pour elle et ainsi, le risque de rechute trop présent. S'éloigner de la drogue passait par une rupture avec Montréal. (Extrait du journal de bord)

Certes, ces pairs ont déjà connu des formes de distanciation avec le monde de la rue car les critères de recrutement du Collectif imposent, par exemple, des éléments de stabilité d'une durée minimale d'un an (arrêt de la consommation par injection, logement stable, parentalité, etc.). Or, cette distanciation est en constante construction et s'inscrit, cette fois, dans la mise en œuvre d'un nouveau rôle qui fait le lien entre les deux mondes. Une expérience de travail qui devient un atout dans une trajectoire professionnelle. C'est ce que raconte U.:

On a appris plein de choses puis aussi la valorisation de travailler, la confiance en soi, etc. [...]. C'est sûr que c'est le parcours qui m’a aidé, si j'avais fait juste le projet des pairs, je serais peut-être pas ici, mais c'est sûr que ça m’a peut-être donné la soif de vouloir faire ça, de vouloir travailler dans le communautaire, aider les gens [...]. C'est moi en premier qui avait dit bon, je veux faire ça [exemple d'intervention témoignage dans les écoles], parler à des jeunes, parler à des plus vieux par rapport à mon expérience, puis finalement, je me le suis fait offrir. (Entrevue, ancien pair, U.)

Il apparaît clairement que la participation au Collectif a contribué à l'intégration biographique de l'expérience de rue des pairs tout en soutenant leur construction identitaire, même si celle-ci ne s'achève pas au terme de leur participation au sein du Collectif $^{8}$. De ce fait, si certains

7. La réalisation des entrevues de la recherche sur la sortie de rue pour les anciens pairs-aidants a coïncidé, comme nous l'avons indiqué dans la partie méthodologique, avec l'organisation de retrouvailles pour l'anniversaire des 10 ans du Collectif. La reprise de contact avec les «anciens» a été conduite par le Collectif, qui mentionnait alors notre étude et demandait l'autorisation de transmettre leurs coordonnées à l'équipe de recherche. De cette manière, nous nous avons pu réaliser cette entrevue téléphonique, cette paire n'ayant pas souhaité venir à l'événement des retrouvailles.

8. Compte tenu du temps de participation variable, pouvant aller jusqu'à deux ans, le départ du Collectif est le plus souvent lié au choix du pair. Dans des circonstances exceptionnelles, par exemple une rechute importante dans la consommation de drogue ou un conflit ouvert avec les autres pairs, le Collectif peut opter pour un renvoi. Même dans ces 
jeunes partent du Collectif quand ils le souhaitent, avec un projet défini, d'autres partent sans avoir un projet réel, mais avec cette expérience dans leur tête et un CV enrichi d'une expérience de travail conventionnelle. Pour certains jeunes, ce travail d'intégration biographique n'est pas toujours évident à réaliser. Si, dans les mots des pairs, le Collectif apparaît comme un «espace», une «coquille», un «cocon» pour travailler cette tension identitaire, tous ne parviennent pas à utiliser ce lieu comme tremplin. Si les échecs de participation au Collectif sont peu nombreux - sur environ 80 jeunes qui sont passés par le Collectif, 3 seulement ont été congédiés et 3 ont abandonné le projet -, tous n'ont pas vécu facilement cette intégration biographique après leur passage dans le Collectif. Néanmoins, la grande majorité l'a utilisée comme une manière de se positionner singulièrement entre la marge et le centre, retenant des valeurs, des attitudes qu'ils associent au monde de la rue qui les emportent dans un processus de sortie de rue, dans d'autres espaces d'affiliation et d'intégration.

On l'a vu, le modèle d'intervention du centre-ville évoque pour plusieurs l'idée d'occasions et d'opportunités. Une sorte de laboratoire où sont canalisés les aptitudes des pairs à aider les autres et leur désir de passer à autre chose. Occasions de prendre un recul face au mode de vie de la rue et à la consommation. Opportunités d'équilibrer le formel et l'informel, la rue et, par exemple, le système de services sociosanitaires. Loutil d'évaluation intégré dans les activités du Collectif «bilan personnel», par exemple, amène le pair à prendre conscience de son influence positive sur ses amis et son entourage en matière de prévention des ITSS et des méfaits de la toxicomanie, mais aussi, il est une manière de revenir sur les réalisations qu'il a accomplies. Cette prise de conscience est stimulante et valorisante pour le pair:

[...] de participer au bilan puis tout ça, ça m’a amené un regard extérieur, externe, sur le projet, [...], un regard que j'avais pas avant. (Entrevue, bilan personnel, S.)

Enfin, la dimension collective, également liée aux dimensions personnelle et professionnelle, façonne le Collectif d'intervention comme une plaque tournante où des jeunes s'engagent, puis passent le flambeau à d'autres jeunes et ainsi de suite. Selon les termes employés par un pair, 
le modèle s'inspire de la vie, comme dans les tribus, c'est-à-dire «du monde qui en aide d'autres à partir de leur vécu et de leur recul». Règle générale, lorsque le pair renvoie à l'apport du Collectif dans son cheminement, les éléments liés au travail d'équipe, au fait d'apprendre à «dealer» avec les autres, même en situation de conflit, à faire des compromis, etc., sont soulignés. Tous avouent qu'il s'agit d'un immense défi, doublé néanmoins d'un atout qui ne manquera pas de leur servir dans la vie et dans un futur emploi:

Pour moi, le projet il représente une étape, moi dans le fond je me sers de ce projet-là pour compenser les études que je suis pas capable d'aller chercher pour aller travailler dans le domaine de l'intervention du travail de rue. (Entrevue, pair, J.)

En somme, le modèle d'intervention par les pairs peut être défini comme un lieu de circulation des savoirs, une sorte d'incubateur toutes portes ouvertes, où des jeunes poursuivent leur cheminement personnel à travers une dynamique d'intervention qui leur permet de redonner à d'autres ce qu'ils ont reçu et ce qu'ils reçoivent encore, jusqu'à ce qu'ils se sentent prêts à un "passage à autre chose», laissant alors leur place à d'autres jeunes. Toutefois, une telle expérience ne peut prendre tout son sens que dans un cadre de reconnaissance, initialement pour le travail du pair lui-même.

\section{Etre reconnu: un nouveau rapport aux autres}

Le positionnement du pair en tant que modèle positif comme exige, de facto, une reconnaissance de ce qu'ils sont et de ce qu'ils font, tant par les jeunes que par les autres acteurs du projet et in fine, par la société en général.

En ce qui a trait aux jeunes de la rue, ceux qui sont visés par l'intervention du Collectif, les mécanismes de reconnaissance passent par le fait que les pairs sont perçus comme proches des jeunes de la rue et crédibles dans le domaine de l'intervention. Voilà comment une des jeunes de la rue nous parle du pair-aidant avec qui elle est en relation:

Pour moi, t'sais elle est comme plus qu'un intervenant. T'sais elle a fait le spectacle avec nous, à la fin ça fait que moi dans ma tête c'était une fille qui trippait le cirque. Pis elle était quand même capable de faire de l'intervention. T'sais nous expliquer des affaires pis toute. (Entrevue, jeune de la rue, C.) 
Dans d'autres circonstances, la reconnaissance vient directement d'une action posée par le pair dans son milieu:

Comme moi, X [une pair-aidante], à un moment donné, j'étais dans la marde, pu d'argent pour payer mon loyer, j'étais pu capable de budgéter puis je capotais ben raide, pu de bouffe, pu rien. Ça fait qu'elle m'a amené chez eux, elle m'a donné de la bouffe, elle m’a montré comment faire un budget. Fa que je lui ai donné du cash, c'est elle qui gérait mon cash un moment donné parce que moi j'étais pu capable. Pis ça les autres intervenants veulent pas faire ça. Mais moi j'savais, si j'ai du cash, je me crinque (injecte) et X, elle a compris que c'est ça qui me fallait. Ça fait que moi j’ai arrêté de me brosser les dents pis tout le kit, puis là, X, elle m'a amené, elle avait vu les gencives, je crachais du sang puis toute. Elle a dit: «Tabarnak ma nana.» J'ai dit: «Oui oui.» Elle a dit: «Viens t'en au CLSC, pourquoi tu y vas pas?» J'ai dit: "J'ai pas le temps d'appeler là, j'ai pas le temps.» J'étais en plein bordel, je venais d'emménager dans mon nouvel appart, j'ai perdu mon appart parce que j'avais un chien. Pis elle m'a aidé avec son truck pour mon frigo pour mon nouvel appart. Pis là en même temps, elle a dit: «Je vais t'arranger ça.» Elle m'a appelé 2 jours après: "T'as un rendez vous au CLSC, dentiste, telle date.» «Estie, t'es cool, merci beaucoup.» Moi je l'aurais pas fait parce que c'est mes dents. J'aurais fait n'importe quoi d'autre sur mon corps, j'aurais appelé moi-même, mais elle le savait ben que pour mes dents j'aurais rien fait. Oui, c'est X, il fallait qu'elle me donne un coup de pied dans le cul, c'est X qui me l'a donné, tu sais. (Entrevue, jeune de la rue, G.)

Cette longue citation permet de situer assez justement le travail du pair-aidant dans sa globalité, dans son humanité, mais aussi dans sa complexité. Pour les jeunes de la rue, la reconnaissance qu'ils ont à l'endroit des pairs-aidants tient, le plus souvent, au fait qu'ils les définissent comme ayant fait une différence dans leur situation de survie ou dans la gestion d'eux-mêmes, des choses de la vie et de leur santé.

Si la reconnaissance du travail des pairs-aidants par les jeunes de la rue est cruciale pour le Collectif et ses membres - rappelons que la réalisation de leur mandat dépend des effets préventifs qu'ils parviennent à concrétiser auprès de ces derniers -, il importe que cette reconnaissance dépasse le monde de la rue. La recherche évaluative que nous avons menée nous a permis de démontrer la contribution des pairsaidants à une articulation singulière de la prévention des ITSS, qui centre les actions autour de la santé plutôt que de la maladie; de la globalité plutôt que la spécificité de certains comportements à risque; autour du souci, en opposition au contrôle, de soi et de son corps (Bellot 
et al., 2006). Cet apport singulier de l'intervention par les pairs au système plus général de prévention ne pourra toutefois survivre sans l'élaboration et le maintien de relations de réciprocité entre le monde de la rue et le monde socio-sanitaire: un cadre de reconnaissance.

La reconnaissance pour les pairs-aidants se vit à travers les relations partenariales qui s'établissent dans le Collectif, entre les pairs et les partenaires du monde socio-sanitaire: des intervenants-superviseurs aux gestionnaires des organismes. En effet, reconnaître l'importance des savoirs expérientiels, appuyer le rôle du pair-aidant dans les ressources et lui attribuer une place à part entière dans les équipes de travail, constituent pour les pairs une preuve essentielle du bien-fondé de leur apport. De l'avis de plusieurs, qu'il s'agisse du lien avec l'intervenant-superviseur, avec la ressource d'appartenance ou avec le groupe des pairs-aidants, chacune de ces composantes est fondamentale, mais l'équilibre entre les trois revêt une importance primordiale. Imaginons, par exemple, que le pair-aidant s'harmonise bien à sa ressource d'appartenance, mais pas au groupe des pairs. Tôt ou tard il devra abandonner le projet. Le même résultat est probable si le pair-aidant s'intègre bien au groupe, mais mal à sa ressource. Les deux situations ont d'ailleurs été vécues dans l'histoire du projet. Qui plus est, elles illustrent bien le partage égalitaire du pouvoir entre le groupe des pairs-aidants et les organismes partenaires.

Cette dynamique égalitaire met en place le socle de rapports sociaux de reconnaissance où les pairs font l'expérience de nouvelles relations avec les adultes et le monde de l'intervention, dans une dynamique de type «démocratie participative». Les rencontres de coordination témoignent de ce travail constant de construction commune des grandes orientations, où chaque acteur a sa place et représente son univers, les pairs comme les autres.

En somme, nous décrivons ici un cadre, une posture d'équilibre, qui permettent de rendre harmonieuses tant l'intégration biographique, l'action préventive, que les relations entre les deux mondes. Or, cet équilibre reste et restera fragile. Comme nous avons pu l'observer en cours d'évaluation, il n'est pas toujours acquis et constitue un défi, une quête continue. Les tendances vers une moindre reconnaissance des pairs-aidants par les partenaires ou, à l'opposé, vers l'autogestion complète par les pairs, sont réelles. Le travail de coordination, le suivi du projet par des évaluations annuelles, la vigilance assurée, notamment par la recherche, mais aussi par des membres fondateurs du Collectif, 
constituent autant d'outils qui permettent de saisir les tensions, d'en débattre collectivement et de maintenir l'équilibre, si tant est que ce principe survit à la mobilité incontournable des acteurs.

Nous avons surtout indiqué, dans nos analyses de l'intégration biographique, les conditions de cette intégration pour dépasser le discours normatif structuré autour de la sortie de la rue. Dans le récit des jeunes, ces conditions sont la construction réflexive de soi pour se définir comme modèle; la production de logiques d'actions incarnant ses propres valeurs; et la reconnaissance des savoirs expérientiels. L'analyse des données des deux recherches nous autorise à avancer que cette dynamique d'intégration biographique est possible. Pourtant, ce cadre de reconnaissance doit pouvoir s'exprimer en dehors du Collectif, afin que les pairs-aidants puissent appréhender la nature de nouveaux rapports sociaux et poursuivre leur cheminement vers «un ailleurs que la rue». Dans ces circonstances, c'est le fait que leur expérience de la rue soit construite comme un savoir et une compétence, au lieu d'un problème, qui permet, dans leur chemin vers «la sortie», d'établir un positionnement identitaire entre la rue et le monde conventionnel. C'est en effet autour de la tension marginalité/conventionnalité que l'intégration biographique de leur expérience de rue et sa reconnaissance constituent un gage pour les pairs, une preuve de leur nouvelle relation au monde, sans pour autant y perdre leurs valeurs et leur authenticité. La situation particulière du Collectif d'intervention par les pairs, qui retient l'expérience de vie comme ressource pour l'intervention, sans viser la «réinsertion», est un lieu privilégié pour lire cette intégration biographique.

Ultimement, les pairs assument leur parcours et créent, à leur image et selon leurs besoins, leur "passage à autre chose», dans la continuité de leurs expériences de rue. Néanmoins, des pairs ont insisté pour dire que le dispositif les a certes aidés, mais qu'il «n'a pas tout fait», ni tout réglé dans leur processus de «sortie» de l'expérience de vie de rue. Ils indiquent ici le lourd travail sur soi qui doit se faire seul et soulignent que le Collectif s'inscrit dans une démarche d'estime de soi et de reconnaissance de son rôle et de sa valeur aux yeux des autres. Évidemment, tout devient plus fort lorsque cette reconnaissance dépasse le Collectif, lorsque le pair part et s'engage dans d'autres expériences:

T'sais partir des pairs, c'est une longue décision. Personne ne les pousse, il faut choisir le bon moment. Ça veut le bon moment pour le pair, pour le groupe, et pour le Collectif. Pour certains, c'est parce qu'ils ont une 
nouvelle job, pour d'autres, c'est parce qu'ils sont prêts à s'aventurer dans d'autres projets, malheureusement, pour une minorité, c'est parce que la drogue est revenue les hanter. Mais tu vois ces retrouvailles [événement], elles montrent ce qu'ils sont devenus et pis, il faut être fiers. Regarde-les, la plupart sont insérés comme on dirait et pourtant, ils ont tous encore leur touche marginale. C'est ça ce qu'ça leur a donné le Collectif, se bricoler une sortie de rue tout en restant soi-même. Pis c'était même pas eux qui étaient visés. Imagine donc comment ce projet est fait fort. (Entrevue, partenaire, $\mathrm{H}$.)

\section{Conclusion}

Une nouvelle exploitation des résultats des deux recherches impose un constat: la question de la sortie de rue ne peut se soustraire, d'une part, à l'intégration biographique de l'expérience de rue par le jeune et ce, au-delà de la rue et, d'autre part, aux enjeux de reconnaissance, mais davantage de non-reconnaissance, qu'ont connus et que connaissent toujours les jeunes en situation de rue (Renault, 2004).

Cet article montre ainsi comment il est possible de dépasser les enjeux normatifs qui encadrent la définition des relations entre pairs, dans le contexte de situations dites déviantes ou délinquantes. La réalité est beaucoup plus complexe que le laisse paraître la position qui diabolise la rue et l'expérience de rue. Certes, il ne s'agit pas non plus de valoriser cette expérience, mais plutôt, comme en témoigne le dispositif présenté, de regarder de manière pragmatique à la fois l'expérience de rue et les manières de soutenir une prévention efficace des difficultés inhérentes à cet espace. Un pari audacieux, qui sous-tend de nombreuses ruptures, la première étant de reconnaître le potentiel de ces jeunes. Lorsque tenu, un tel pari permet à tous d'y gagner.

\section{Références}

Backett-Milburn, K.; Wilson, S. (2000). Understanding peer education : insights from a process evaluation. Health Education Research, 15 (1), 85-96.

Bajoit, G. (2003). Le changement social: approche sociologique des sociétés occidentales contemporaines. Paris, Armand Colin.

Bajoit, G. (2000). Qu'est-ce que la socialisation? Jeunesse et société, in Bajoit G., Digneffe F., Jaspard J-M., Nollet de brauwere, Q. Jeunesses et société: la socialisation des jeunes dans un monde en mutation. Bruxelles, De Boeck Université, Ouvertures sociologiques, 19-41. 
Baron, S. (2008). Street youth, unemployment, and Crime: is it that simple? Using general strain theory to untangle the relationship? Criminologie, vol. 50, n. 4, 399-434.

Bellot, C. (2001). Le monde social de la rue: expériences des jeunes et pratiques d'intervention à Montréal, Thèse de doctorat, École de criminologie, Université de Montréal.

Bellot, C. (2005). La diversité des trajectoires de rue des jeunes à Montréal, in Brunelle N, Cousineau, M-M., Trajectoires déviantes des jeunes: éclairage des recherches qualitatives, Presses de l'Université du Québec, 71-93.

Bellot, C.; Rivard J. (2007). L'intervention par les pairs: un enjeu pour la reconnaissance in Baillergeau, E.; Bellot, C. Transformations de l'intervention sociale. Entre innovation et gestion des nouvelles vulnérabilités? PUQ, 173-202.

Bellot C., Rivard J., Mercier C., Fortier J., Noël V., CIMON M-N. (2006). Le projet d'intervention par les pairs auprès des jeunes de la rue du centre-ville de Montréal: une contribution majeure à la prévention, Rapport de recherche au FQRSC, Collectif des Pairs, Montréal. http://cactusmontreal.org/fr/pairaidants.html.

Bergier, B. (2000). Les affranchis, Paris, L'Harmattan.

Bertaux, D. (1997). Les récits de vie, Paris, Nathan.

Caputo, T.; Weiler R.; Anderson, J. (1997). Étude sur le style de vie de la rue, Ottawa, Santé Canada, Bureau de l'alcool, des drogues et des questions de dépendance.

Chazy, O. (1999). Les enfants et les jeunes à la rue en France, in Programme pour l'enfance. Groupe d'experts sur les enfants en errance, Conseil de l'Europe, Strasbourg, 30-33.

Chobeaux, F. (196). Les nomades du vide, Arles, Actes Sud.

Colombo, A. (2001). Analyse du processus de changement de mode de vie chez les jeunes de la rue à Montréal, Mémoire de Licence, Travail social et politiques sociales, Fribourg, Université de Fribourg.

Côté, M-M. (1988). Les jeunes de la rue à Montréal. Une étude d'etbnologie urbaine, Thèse de doctorat, Département d'anthropologie, Montréal, Université de Montréal.

Delory-Momberger, C. (2004). «Introduction», L'orientation scolaire et professionnelle [En ligne], 33/4 | 2004, mis en ligne le 28 septembre 2009, Consulté le 29 mars 2010. URL: http://osp.revues.org/index261.html

Delory-Momberger, C. (2004). «Biographie, socialisation, formation», L'orientation scolaire et professionnelle [En ligne], 33/4 | 2004, document 4, mis en ligne le 28 septembre 2009, Consulté le 29 mars 2010. URL: http://osp. revues.org/index 251. html

Direction de la santé publique (1998). Le «Défi de l'accès» pour les jeunes de la rue. Montréal. Régie régionale de la Santé et des Services sociaux de MontréalCentre.

Dubet, F. (1995). La sociologie de l'expérience. Paris: Seuil.

Ehrenberg, A. (1996). L'individu incertain. Paris: Hachette Littérature. 
Fontaine, N; Vitaro, F. (2006). L'utilisation de pairs prosociaux dans les programmes d'intervention auprès des jeunes en difficulté d'adaptation. Revue de psychoéducation, 35, 1, 11-42.

Godbout, J.T. (1992). L'esprit du don, Paris, La découverte.

Goyette, M.; Bellot, C.; Panet-Raymond, J. (2004). Le projet solidarité jeunesse. Dynamiques partenariales et insertion des jeunes en difficulté, Québec, Presses de l'Université du Québec.

Greissler, E. (2007). Entre marginalité et conformité: la construction identitaire des jeunes de la rue. Mémoire de maîtrise, École de service social, Université de Montréal.

Hagan, J.; McCarthy, B. (1998). Mean Streets: Youth Crime and Homelessness. Cambridge: Cambridge University Press.

Karsz, S. (2004). Pourquoi le travail social? Définition, figures, clinique. Paris, Dunod.

Lahire, B. (1998). L'Homme pluriel. Les ressorts de l'action. Paris, Nathan.

Lamoureux, J. (2001). Marges et citoyenneté, Sociologie et sociétés, XXXIII, 2, 29-47.

Lanctôt, N.; Cernkovich, S.; Giordano, P.C. (2007). Delinquent Behavior; Official Delinquency and Gender : Consequences for Adulthood Functioning and Well-Being. Criminology, 45, 1, 131-158.

Lucchini R. (1999). L'enfant de la rue: carrière, identité et sortie de rue, Fribourg, Presses universitaires de Fribourg.

Martucelli, D. (2005). Les trois voies de l'individu sociologique. EspacesTemps.net, Textuel, 08.06.2005 http://espacestemps.net/document1414.html, consultation 15 mars 2010

Milburn, K. (1995). A critical review of peer education with young people with special reference to sexual bealth. Health Education Research, 10, 407-420.

Morizot, J.; Le Blanc, M. (2000). Le rôle des pairs dans l'émergence et le développement de la conduite délinquante: une recension critique des écrits. Revue canadienne de psychoéducation, 29, 87-118.

Parazelli, M. (1998) Aller voir ailleurs si j’y suis... Les trajectoires géosociales des jeunes de la rue québécois, Agora, 13, 71-86.

Parazelli, M. (2002). La rue attractive. Parcours et pratiques identitaires des jeunes de la rue, Québec, Les Presses de l'Université du Québec.

Parazelli, M. (2003). La marginalité serait-elle normale? in ROUSSEAU V., Ed., Indiscipline et marginalité. Actes du colloque, Montréal, Société des arts indisciplinés, 67-87.

Parazelli, M.; Colombo, A. (2006). Intervenir aux marges de la citoyenneté. Une application du dispositif Mendel adapté au contexte des jeunes de la rue à Montréal, Nouvelles pratiques sociales, 18, 2, 88-109.

Pattegay, P. (2003). L'actuelle construction, en France, du problème des jeunes en errance. Analyse critique d'une catégorie d'action publique. Déviance et société, 3, 25, 257-277.

Proulx, M-C.; Gratton, F. (2006). Processus suivi par des pairs aidants lors de leur expérience auprès d'adolescents suicidaires: une étude exploratoire. Santé Mentale au Québec, 31,1, 145-168. 
Renault, E. (2004). L'expérience de l'injustice. Reconnaissance et clinique de l'injustice, Paris, La Découverte.

Rivard, J. (2004).Des pratiques autour des jeunes/enfants des rues: une perspective internationale, Nouvelles pratiques sociales, Volume 17, numéro 1, 2004, 126-148

Roy, É; Haley, N. ; Leclerc, P.; Lemire, N. ; Boivin, J-F. ; Frappier, J-Y. ;Claessens, C. (2000). Prevalence of HIV infection and risk behaviours among Montreal street youth. International Journal of STD and AIDS, 11, 241-247.

Roy, É. ; Lemire, N.; Haley, N. ; Boivin, J-F.; Frappier, J-Y.; Claessens, C. (1998). Injection Drug Use Among Street Youth A Dynamic Process. Canadian Journal of Public Health, vol 89, 40, 239-240.

Sciacca, J-P.; Black, D.R. (1996). A critical review of peer education with young people with special reference to sexual health: are we throwing the baby out with the bath water?. The Peer Facilitator Quarterly, 14, 17-19.

Shériff, T. (1999). Le trip de la rue. Parcours initiatiques des jeunes de la rue, Tome I, Beauport, Centre jeunesse de Québec.

Tremintin, J. (1999). Comment les jeunes errants mettent le travail social en difficulté, Lien social, www.lien-social.com/archives/dossiers/471a480/4732.htm.

Yin, R. (1994). Case study research: Design and methods, Beverly Hills, CA: Sage Publishing.

ABSTRACT • Little is still known about street youth leaving the street, i.e. their street exit. In this article, we aim to address the question of street exit by using two studies, each of them with the same topic: a peer-intervention with street youth (Collectif d'intervention par les pairs). We intend to understand how the Collectif des pairs' frame of intervention influences peer tutors' life trajectories. The Collectif des pairs gives peer tutors a new role: it enables them to view the street from a more distant perspective while remaining close to the street, thus giving them the opportunity to engage in mainstream intervention. Nonetheless, beyond their participation, important stakes of recognition and non-recognition of street youth remain.

KEYWORDS - Street youth, Peer-intervention, trajectories, identity, deviance/marginality.

RESUMEN - Son pocos los estudios científicos sobre los jóvenes que dejan la calle. En este artículo nos proponemos abordar el asunto analizando los resultados de dos estudios que realizamos sobre un grupo de intervención de pares, el Collectif d'intervention par les pairs. El objetivo es analizar los aportes de este marco de intervención en la trayectoria de los jóvenes. Al participar en este tipo de intervención, los jóvenes que han vivido en la calle se colocan en una nueva función que les permite al mismo tiempo tomar distancia y mantener la cercanía con el mundo de la calle, en una oportunidad real de formar parte del mundo de la intervención convencional. Sin embargo, más allá de esta intervención, ¿qué decir de los aspectos de reconocimiento y falta de reconocimiento que viven los jóvenes de la calle?

PALABRAS CLAVE - Jóvenes de la calle, dejar la calle, intervención en medio abierto, ayuda de pares, trayectoria, identidad, marginalidad. 